\title{
Sensitivity of Sub-Bandgap External Quantum Efficiency Measurements of Solar Cells under Electrical and Light Bias
}

\author{
Stefan Zeiske, Christina Kaiser, Paul Meredith, and Ardalan Armin*(i) \\ Sustainable Advanced Materials (Sêr-SAM), Department of Physics, Swansea University, Singleton Park, Swansea, SA2 8PP Wales, \\ United Kingdom
}

\section{Supporting Information}

ABSTRACT: The measurement of the external quantum efficiency (EQE) for photocurrent generation at photon energies below the bandgap of semiconductors has always been an important tool for understanding phenomena such as charge photogeneration via tail and trap states. The shape of the subgap EQE can also reveal the subtle but important physics of inter- and intramolecular states that lay at the heart of charge photogeneration in molecular systems such as organic semiconductors. In this work, we examine the influence of optical and electrical noise on the sensitivity of EQE measurements under different electrical and optical bias conditions and demonstrate how to enhance the dynamic range to an unprecedented $>100 \mathrm{~dB}$. We identify and study several apparatus-and-device-related factors limiting the sensitivity including: the electrical noise floor of the measurement system; flicker and pick-up noise; probe light source stray light; the photon noise of the light bias source; the electrical noise of the voltage bias source; and the shunt-resistance-limited thermal and electrical shot noise of the device. By understanding and minimizing the influence of these factors we are able to detect EQE signals derived from weak subgap absorption features in both organic and inorganic solar cell systems at photon energies well below their bandgaps.

KEYWORDS: solar cells, sensetive external quantum efficiency, trap states, charge transfer states, sub-bandgap absorption, photocurrent spectroscopy
S ensions ensitive measurements of photocurrent in solar cells and photodetectors is becoming increasingly important for achieving a better understanding of the mechanism of light absorption and charge generation. This is particularly the case for semiconductors (such as the organics) which can be considered as molecular with a high degree of disorder. Accurate measurement of photocurrent, and more particularly the external quantum efficiency or EQE, below the semiconductor optical bandgap is challenging because the absorption coefficient is orders of magnitude less than abovegap. In most crystalline inorganic and perovskite semiconductor devices (such as solar cells and photodetectors) the subgap EQE reveals information about the sub-band states such as traps ${ }^{1,2}$ and energetic disorder. ${ }^{3-5}$ In the equivalent organic semiconductor devices, the subgap EQE is often used to investigate so-called charge transfer (CT) states. $^{6-8}$ The contribution of these subgap states to the photocurrent also plays an important role in the determination of the radiative limit of the open-circuit voltage. ${ }^{9}$

In several seminal works, the subgap photocurrent has been determined using Fourier transform photocurrent spectroscopy as a fast and sensitive method. ${ }^{10-12}$ This technique can reveal the contribution of photoactive species to the photocurrent with absorption coefficients orders of magnitude lower than direct band-to-band transitions. These and related EQE measurements have also proven to be sensitive enough to quantify the contribution of subgap states such as the CT states in organic semiconductors ${ }^{7}$ and, thus, have made a significant impact on our understanding of the mechanism of charge generation in organic optoelectronic devices and particularly the physics of CT states. For example, by measuring the EQE sensitively in combination with photothermal deflection spectroscopy (PDS) Vandewal et al. have shown relaxed CT states (directly excited by low energy photons) can contribute to the photocurrent as efficiently as those CT states populated via singlet excitons with energies in excess of the CT state energy. ${ }^{7}$ Sensitive EQE measurements are now often used in the literature for determination of CT state energies as well as their reorganization energies. ${ }^{13,14}$ Absorption coefficients and cross sections of CT states have also been inferred from sensitive EQE measurements. ${ }^{15}$ All of these parameters are crucial for understanding the open-circuit voltage of organic solar cells and to determine nonradiative voltage losses versus CT state energy in conjunction with electroluminescent measurements via the detailed balance principle. $^{16}$

Received: October 21, 2019

Published: December 9, 2019 


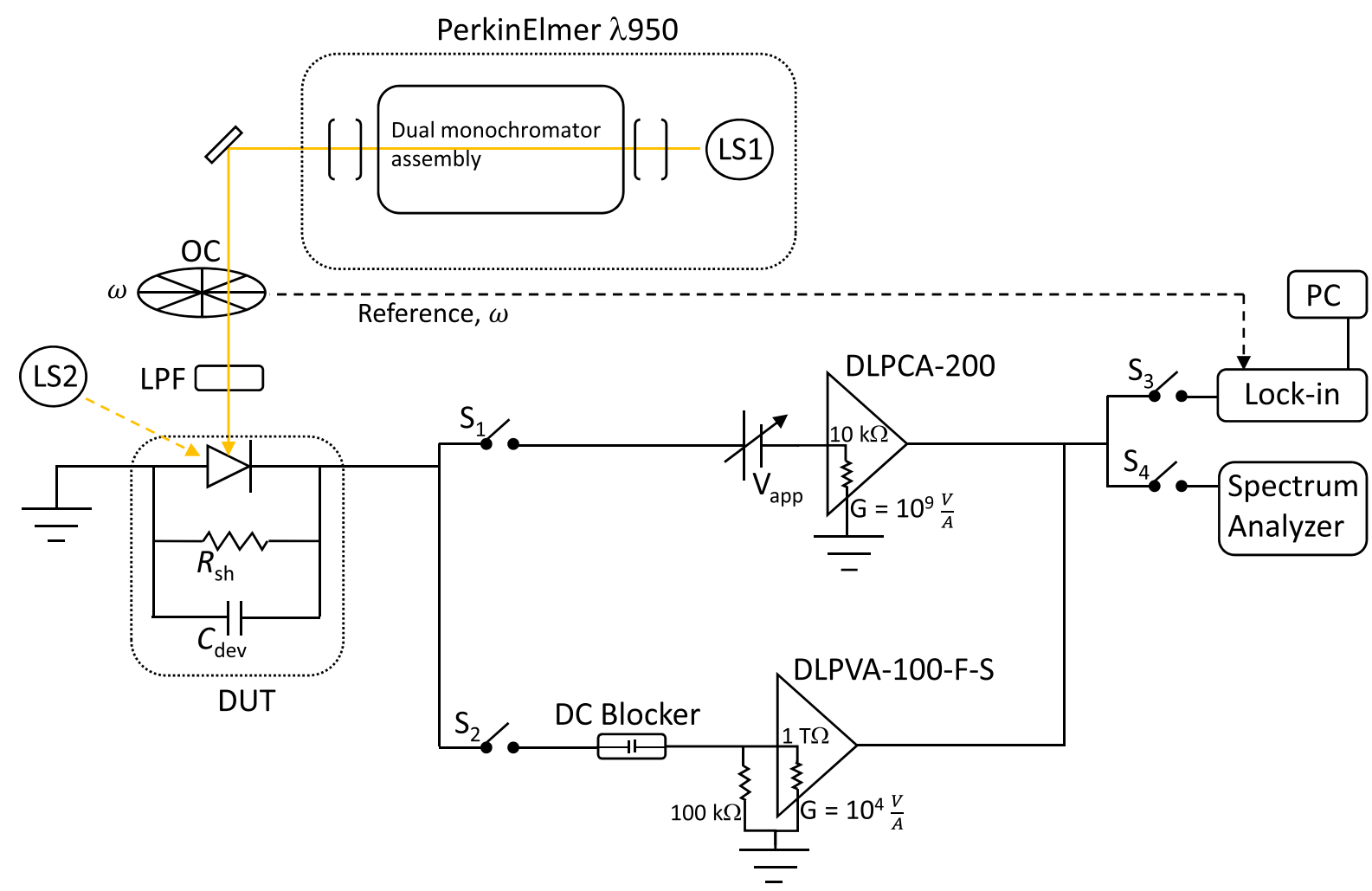

Figure 1. Schematic of the experimental setup for sensitive external quantum efficiency (EQE) measurements using a 2950 (PerkinElmer) spectrophotometer. An optical chopper (OC) is used for modulating the monochromatic light source. Prior to focusing the light on the device under test (DUT), different long pass filters (LPF) can be used to filter out parasitic stray light and harmonics of the monochromator. Using switch S1, the current preamplifier amplifies the photocurrent signal and allows for biasing the DUT using its internal low noise voltage source. Switch S2 optionally allows for the measurement of EQE under open-circuit conditions using the high input impedance (1 T $\Omega$ ) voltage preamplifier in series with a DC blocker and a $100 \mathrm{k} \Omega$ resistor (corresponds to a gain of $100 \mathrm{~dB}$ ). A lock-in amplifier is used to read the amplified AC photocurrent signal of the DUT at a specified electrical bandwidth. Switch $S_{4}$ enables the noise spectral density (NSD) measurements via a spectrum analyzer. Optionally, an additional light source (LS2) with variable intensity can be used for measurements of the EQE sensitively under light bias.

The importance of measuring EQE spectra sensitively is thus increasingly appreciated and this motivated our current study. In this regard, the aim of the present work is to push the boundaries of EQE sensitivity by understanding what fundamentally limits its measurement and thus how to improve it. To achieve this, we have used a commercial spectrophotometer with a double monochromator architecture as an ultrastable wide spectral range light source and examined inorganic and organic solar cells as model test systems to probe noise and sensitivity of the EQE measurements. We demonstrate how electrical and optical noise limits the sensitivity of EQE measurements and propose a simple means to reduce them and to increase the minimum detectable EQE. This is especially important for measuring EQE sensitively under white light or electrical bias, where the device electrical shot noise or light bias optical shot noise are limiting factors. Finally, we show that with an apparatus limit of $100 \mathrm{~dB}$ (equivalent to a minimum detectable EQE on the order of $10^{-10} \mathrm{~Hz}^{-1 / 2}$, corresponding to photocurrents smaller than a $\mathrm{fA}$ ) EQEs can be measured down to the thermal noise limit at short-circuit, the electrical shot noise limit under electrical bias, and the photon shot noise limit for white-light biased devices.

\section{BASIC CONSIDERATIONS FOR RESPONSIVITY, SENSITIVITY, AND MINIMUM DETECTABLE EQE}

The responsivity, $R$ is defined as the first derivative of the device photocurrent $I$ with respect to incident light power $L$ :

$$
R=\frac{\mathrm{d} I}{\mathrm{~d} L}=\frac{\lambda q}{h c} \cdot \frac{\phi_{\mathrm{e}}}{\phi_{\mathrm{ph}}}
$$

where $\lambda$ is the excitation wavelength, $q$ is the elementary charge, $h$ is the Planck constant, and $c$ is the speed of light in vacuum. Here, $\phi_{\mathrm{e}}$ denotes the flux of extracted photogenerated electrons, whereas $\phi_{\mathrm{ph}}$ denotes the flux of incident photons of energy $\frac{h c}{\lambda}$. The ratio of $\phi_{\mathrm{e}}$ and $\phi_{\mathrm{ph}}$ is then defined as the external quantum efficiency (EQE):

$$
\mathrm{EQE}=\frac{\phi_{\mathrm{e}}}{\phi_{\mathrm{ph}}}=\frac{h c}{q \lambda} \cdot R(\lambda)
$$

An important quantity characterizing an EQE measurement system and its ability to detect weak EQE signals sensitively over several orders of magnitude is the dynamic range (DR) defined as the ratio of the highest $\left(\mathrm{EQE}_{\max }\right)$ and lowest $\left(\mathrm{EQE}_{\text {min }}\right)$ that is detectable above the noise floor. Assuming $\mathrm{EQE}_{\max }$ to be 1 (i.e., in absence of any gain), the $\mathrm{DR}$ is then ultimately given by $\mathrm{EQE}_{\min }$ : 


$$
\mathrm{DR}=10 \cdot \log \left(\frac{1}{\mathrm{EQE}_{\min }}\right)=10 \cdot \log \left(\frac{q \lambda}{h c} \cdot \frac{L(\lambda)}{I_{\text {noise }}}\right)
$$

where $I_{\text {noise }}\left(=I_{\mathrm{NSD}} \sqrt{\Delta f}\right.$, where $I_{\mathrm{NSD}}$ is the noise current spectral density and $\Delta f$ the electrical bandwidth) is the device average noise current. While most conventional solar cells are designed to achieve the highest EQE in the visible (Vis) wavelength regime, $\mathrm{EQE}_{\text {min }}$ will be typically located at longer wavelengths (i.e., at photon energies below the bandgap) in the nearinfrared (NIR). Subsequently, achieving a high DR (low $\left.\mathrm{EQE}_{\text {min }}\right)$ requires in the first instance a light source with particularly high power in the NIR and second, a low noise current $I_{\text {noise }}$. We note that $I_{\text {noise }}$ may be frequency-dependent if flicker noise is present, and at large enough frequencies, it is determined by the thermal noise (dependent on the device shunt resistance) and the shot noise. ${ }^{17-20}$ From eq 2, it follows that the noise equivalent $\mathrm{EQE}$ is given by

$$
\mathrm{EQE}_{\mathrm{NE}}=\frac{h c}{q \lambda} \cdot \frac{I_{\mathrm{NSD}} \cdot \sqrt{\Delta f}}{L}
$$

We note that, as a consequence of this simple equation for $\mathrm{EQE}_{\mathrm{NE}}$, every experimentally measured $\mathrm{EQE}$ signal ( $\mathrm{EQE}_{\text {exp }}$ ) can be assigned to either a real signal above the noise level $\left(\mathrm{EQE}_{\exp }>\mathrm{EQE}_{\mathrm{NE}}\right)$ or noise $\left(\mathrm{EQE}_{\exp } \leq \mathrm{EQE}_{\mathrm{NE}}\right)$.

\section{RESULTS AND DISCUSSION}

Sensitive EQE Experimental Details. Figure 1 shows a schematic of the system for our high sensitivity EQE measurements. In the apparatus, a commercial, high-performance spectrophotometer $\lambda 950$ (PerkinElmer), conventionally used for reflection, transmission and absorption measurements, acts as the (probe) light source. A combined deuterium and tungsten halogen lamp (LS1) provide an extended spectral wavelength regime from $175 \mathrm{~nm}$ up to $3300 \mathrm{~nm}$. Several different optical components, such as filters, mirrors and lenses, together with two double holographic grating monochromators providing a spectral output width of $5 \mathrm{~nm}$ in VIS and $20 \mathrm{~nm}$ in NIR (limited by the chosen slit width) are used to provide an ultralow stray light source with high temporal stability and flexibility to control spot size and polarization. The output light from the spectrometer source is physically chopped by a multiblade chopper wheel (OC) at frequency $f=\frac{\omega}{2 \pi}$ (Thorlabs MC2000B) and different long pass filters (LPF, Edmunds Optics OD4) were used to filter out remaining, parasitic stray light. Switch S1 allows for the detection of the photocurrent signal with a lock-in amplifier (Stanford Research Systems, SR860) passing through a low noise preamplifier (FEMTO, DLPCA-200) with variable gain (up to $10^{9} \mathrm{VA}^{-1}$ at low noise) and integrated low noise voltage source allowing for adjustable bias voltages to be applied $( \pm 10 \mathrm{~V})$. For EQE measurements under open-circuit conditions, switch S2 enables the use of a high input impedance ( $1 \mathrm{~T} \Omega$ ) voltagepreamplifier (FEMTO, DLPVA-100-F-S) with variable gain (up to $80 \mathrm{~dB}$ ) in series with the device under test (DUT). A DC blocker (Thorlabs, EF500) is used to maintain the opencircuit condition when light bias is used. This component is not blocking at the frequency $f(\gg 1 \mathrm{~Hz})$ so that the total DC current is zero (i.e., open circuit) but the AC component can be measured. The $100 \mathrm{k} \Omega$ resistor in parallel with the voltage preamplifier is used to regulate the input impedance of this preamplifier to reduce the $R C$-time constant of the circuit. The lock-in amplifier, providing integration times (i.e., electrical bandwidths) from $1 \mu \mathrm{s}\left(10^{6} \mathrm{~Hz}\right)$ up to $30 \mathrm{ks}\left(\sim 3.33 \times 10^{-5}\right.$ $\mathrm{Hz}$ ) measures the preamplified photocurrent of the DUT. Here, the OC is used as an external reference source for the $\mathrm{AC}$ modulation frequency. For EQE measurements under light bias an additional (pump) light source (LS2) with variable output power can be used to illuminate the DUT. For the calibration process a Newport NIST-calibrated silicon 818-UV (for wavelengths between 200 and $1100 \mathrm{~nm}$ ), germanium 818IR (for wavelengths between 780 and $1800 \mathrm{~nm}$ ), and Thorlabs indium gallium arsenide S148C (for wavelengths between 1200 and $2500 \mathrm{~nm}$ ) photodiode sensor were used. A resolution and video bandwidths of $1 \mathrm{~Hz}$ were utilized for measurements of the NSD using a Keysight Spectrum Analyzer N9010B (SA) operating under AC-coupling.

Noise Equivalent EQE. In order to optimize the EQE apparatus and achieve the large DRs needed to detect weak signals at photon energies well below the bandgap, identification of optical and electrical noise sources is of the utmost importance. The electrical noise of the EQE apparatus was examined by measuring the NSD of the preamplifier at the highest gain of $10^{9} \mathrm{VA}^{-1}$ as a function of frequency (see Figure $2 a)$. The noise level determines the apparatus limit of the EQE measurement when an ideal device (with noise level smaller than the preamplifier) is used. In this figure, the vertical, dashed line marks the frequency of $273 \mathrm{~Hz}$, at which the EQE measurements were performed. Importantly, the frequency of $273 \mathrm{~Hz}$ consciously avoids the flicker noise at very low frequencies $(<10 \mathrm{~Hz})$ (see Figure $\mathrm{S} 1 \mathrm{a}-\mathrm{c}$ ) and the main hum noise peaks at $50 \mathrm{~Hz}$ and multiples (both flicker and hum reduce at higher frequencies). The frequency of the measurement must clearly be chosen to be different to the hum noise peaks. Failing to do so will result in a substantial increase to the background noise of the measurement which cannot be improved by lowering the electrical bandwidth as the hum noise peaks will then appear as parasitic "signal" with constant phase such that they cannot be averaged out by longer integration times. However, one should also be aware that the choice of frequency is not only limited by the technical aspects of the EQE system (e.g., mechanical limitations of the chopper blade or noise peaks of the preamplifier), but also by the underlying physical processes of the DUT, such as charge transport and $R C$-time of the device/circuit as well as trapping and detrapping lifetimes of charge carriers within the active layer. Therefore, higher frequencies greater than the cutoff frequency of the device/circuit combination must additionally be avoided. Failing to do so will result in an underestimation of the EQE. As shown in Figure 2a, a preamplifier NSD of $1.62 \times$ $10^{-15} \mathrm{AHz}^{-1 / 2}$ (at gain $10^{9} \mathrm{VA}^{-1}$ ) at a frequency of $273 \mathrm{~Hz}$ (cf. Figure 2a, vertical dashed line) was measured with the Spectrum Analyzer under dark conditions using an electrical bandwidth of $1 \mathrm{~Hz}$. Figure $2 \mathrm{~b}$ shows the output light power $L$ of the $\lambda 950$ measured with calibrated photodiodes. The EQE is then calculated from eq 4 and shown as the dashed curve. This represents the fundamental limit of the apparatus at an electrical bandwidth of $1 \mathrm{~Hz}$. As shown in Figure $2 \mathrm{~b}$, a minimum $\mathrm{EQE}_{\mathrm{NE}}$ of approximately $1.14 \times 10^{-10} \mathrm{~Hz}^{-1 / 2}$ was achieved at $1200 \mathrm{~nm}$, while an EQE of $2.15 \times 10^{-9}$ was obtained at $2500 \mathrm{~nm}$. It is important to note that the noise equivalent $\mathrm{EQE}\left(\mathrm{EQE}_{\mathrm{NE}}\right)$ ultimately depends on the electrical bandwidth (cf. eq 4), and subsequently, one has to decrease the electrical bandwidth in order to reduce the total noise, 
a)

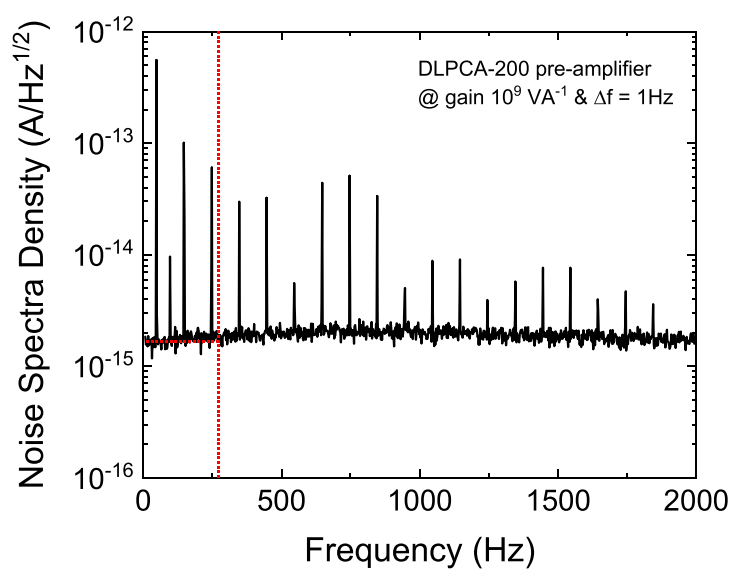

b)

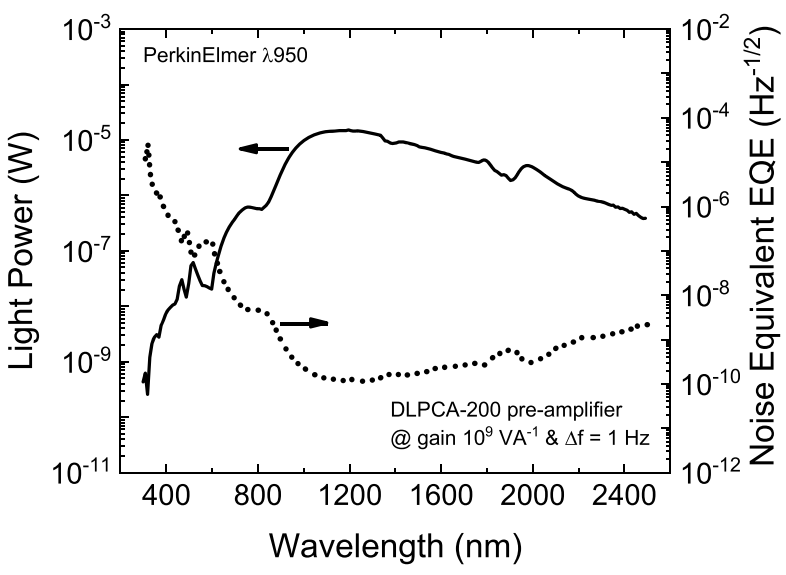

c)

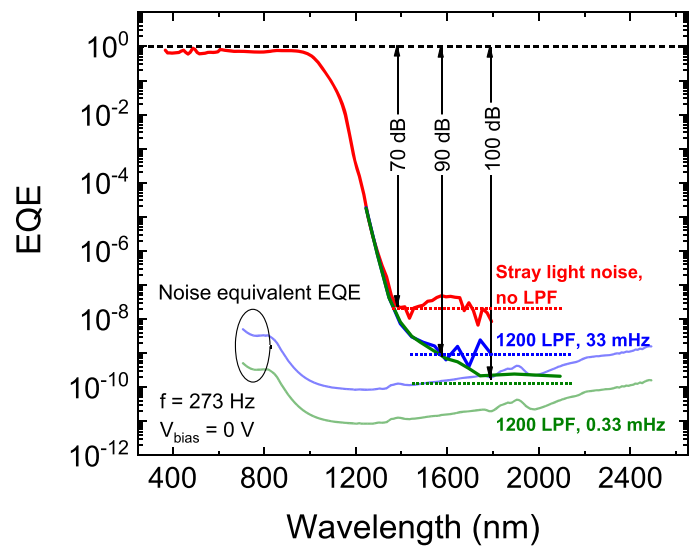

Figure 2. (a) Noise spectral density (NSD) of the preamplifier (PA) plotted as a function of frequency. The vertical dashed line marks the light chopping frequency of $273 \mathrm{~Hz}$, at which the sensitive external quantum efficiency (EQE) measurements are performed. Flicker noise is dominant at very low frequencies $(<10 \mathrm{~Hz})$ and hum noise peaks at harmonics of $50 \mathrm{~Hz}$ are evident. (b) Output power of the $\lambda 950$ spectrophotometer (solid line) measured with calibrated silicon, germanium, and indium gallium arsenide photodiodes and calculated apparatus noise equivalent $\mathrm{EQE}$ spectrum of the current preamplifier (dotted line) representing the minimum detectable EQE spectrum of the apparatus at an electrical bandwidth of $1 \mathrm{~Hz}$. (c) EQE spectrum of a calibrated silicon photodiode. The vertical dashed line marks an EQE of 1 (100\%). When using the $\lambda 950$ as a light source only parasitic stray light (i.e., optical noise) limits the minimum detectable EQE to $10^{-7}$ (solid red line), while the thermal noise limit of the photodiode is at the level of $10^{-9}$ at this electrical bandwidth (dotted blue line), respectively. The use of an additional $1200 \mathrm{~nm}$ long pass filter eliminates the parasitic stray light leading to a minimum
Figure 2. continued

detectable EQE of $10^{-9}$ (green solid line). By lowering the electrical bandwidth by a factor of 100 down to $0.33 \mathrm{mHz}$, the electrical noise reduces 10 times to $10^{-10}$ (dotted green line) resulting in meaningful EQE values as low as $10^{-10}$ (solid green line).

measure a lower EQE and thus achieve a higher DR. This, of course, slows down the speed of the measurement.

We highlight that the total EQE measurement time depends on both electrical bandwidth and (wavelength) step size. For large EQE signals (typically at photon energies above the bandgap) the electrical bandwidth (integration time constant) can be increased (decreased) to $1 \mathrm{~Hz}(1 \mathrm{~s})$, while for EQE signals below the bandgap, we decreased the electrical bandwidth to much lower values, down to $0.33 \mathrm{mHz}$, corresponding to time constants up to $3 \mathrm{ks}$. The measurement time is defined by the relaxation time of the lock-in, which is typically four times larger than the time constant. Subsequently, a total measurement time for a highly sensitive EQE measurement over a broad wavelength regime (i.e., from 300 $\mathrm{nm}$ up to $2500 \mathrm{~nm}$ ) can easily take up to $24 \mathrm{~h}$ to complete.

Sensitive EQE of Inorganic Devices. Having understood and characterized the apparatus-limited $\mathrm{EQE}_{\mathrm{NE}}$, we then investigated a crystalline silicon photodiode as a DUT (see Figure 2c). The EQE measurements were performed at a short-circuit of an electrical bandwidth of $33 \mathrm{mHz}$. The $\mathrm{EQE}_{\mathrm{NE}}$ is calculated from the noise spectral density of the silicon photodiode (see Figure S1a) and plotted in the same panel as a dotted curve. When using the $\lambda 950$ as a light source only (i.e., without additional long pass filters), the experimentally obtained $\mathrm{EQE}_{\exp }$ (cf. solid, red line in Figure 2c) exhibits an apparent sub-bandgap plateau with an EQE of $10^{-7}$. Using an additional $1200 \mathrm{~nm}$ long pass filter, the aforementioned plateau disappears and the $\mathrm{EQE}_{\exp }$ agrees with the thermal-noiselimited $\mathrm{EQE}_{\mathrm{NE}}$ at long enough wavelengths. This implies that, in the absence of the long pass filter, the minimum detectable EQE was limited by the optical (parasitic stray light) noise of the monochromator which in this case was on the order of -70 $\mathrm{dB}$ relative to the main monochromator signal. To further improve the sensitivity, a $100 \times$ lower electrical bandwidth of $0.33 \mathrm{mHz}$ was used, resulting in an $\mathrm{EQE}_{\mathrm{NE}}$ at $1800 \mathrm{~nm}$ of approximately $10^{-10}$ (cf. solid, green line in Figure $2 \mathrm{c}$ ), and a DR of $100 \mathrm{~dB}$ was thus achieved. The photocurrent generated by the DUT at $1800 \mathrm{~nm}$ at the output power of our monochromator was smaller than a fA measured at extremely small electrical bandwidth.

These ultrasensitive EQE measurements obtained through minimizing the electrical and optical noise allowed for the detection of photocurrent at photon energies well below the bandgap of the crystalline silicon photodiode. As shown in Figure $2 c$, deep trap states are revealed in the EQE spectrum at wavelengths above $1400 \mathrm{~nm}$ (photon energies smaller than $0.89 \mathrm{eV}$ ). We then examined the EQEs of a crystalline (c-) (part number KXOB22-12X1, IXYS), polycrystalline (poly-) (JY03264, Shenzhen Yibai Network Technology Co., Ltd.), and hydrogenated amorphous (model number JY03264) (a-) silicon solar cell, as well as a germanium (Ge-) photodiode (818-IR, Newport). These measurements likewise revealed similar subgap absorption features at photon energies well below the constituent semiconductor bandgaps (cf. Figure 3). A trap-induced tail is revealed in the EQE spectra of $\mathrm{c}-\mathrm{Si}$ and poly-Si solar cells and c-Si photodiode at extremely low 


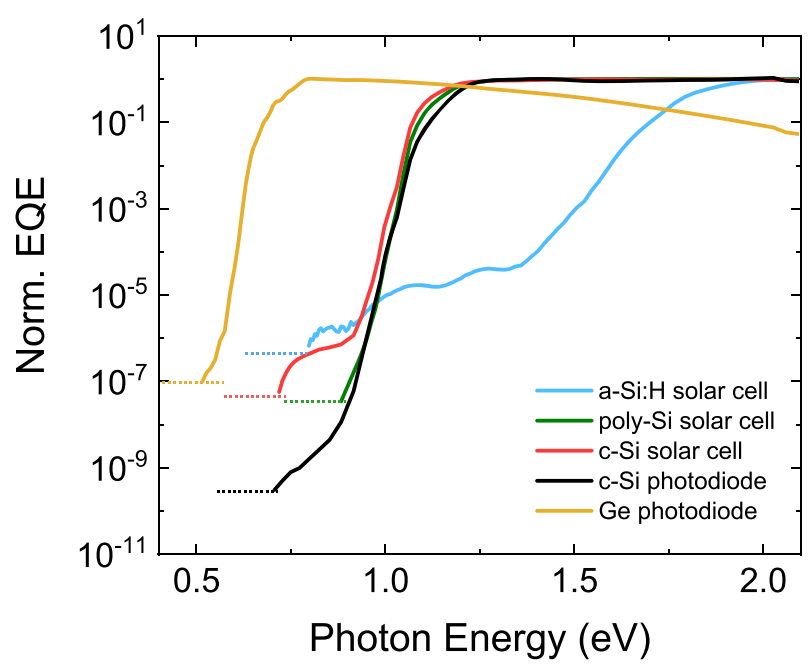

Figure 3. Normalized external quantum efficiency (EQE) of a crystalline silicon (c-Si), a polycrystalline silicon (poly-Si), and a hydrogenated amorphous silicon (a-Si:H) solar cell as well as a silicon and germanium photodiode sensor plotted as a function of photon energy (colored solid lines). Dotted lines mark the respective noise floors (i.e., $\mathrm{EQE}_{\text {exp }} \leq \mathrm{EQE}_{\mathrm{NE}}$ ) for each device obtained at the corresponding electrical bandwidth (varied between 33.33 and 0.33 $\mathrm{mHz}$ ).

responsivities ( -60 to $-80 \mathrm{~dB}$ relative to the above-bandgap EQE). One can also naturally see that in the a-Si:H solar cell the band-edge is much broader than that in c-Si and poly-Si solar cells/photodiodes due to the intrinsic and well-studied energetic disorder. The trap-induced tail of the EQE in a-Si:H is considerably stronger than that of c-Si and poly-Si with three distinct sub-bandgap peaks due to charged-traps and defect states. $^{21}$

Sensitive EQE of Organic Solar Cells under Electrical Bias. Next, we examined a solution processed organic solar cell which often exhibit a larger noise current due to their disorder and structural defects. As mentioned above, sensitive EQE measurements are conventionally used to investigate the contribution of various inter- and intramolecular states in organic solar cells. ${ }^{22}$ Here, the current (voltage) dependent shot noise of the devices can strongly limit the sensitivity of the EQE under electrical bias. Under the short circuit condition, the noise current is limited by the device shunt resistance. ${ }^{23,24}$ Under short-circuit conditions the (thermal) noise current $i_{\text {noise }}$ is inversely proportional to the shunt resistance $R_{\text {shunt }}$ of the device via $i_{\text {noise }}=\sqrt{\frac{4 k_{\mathrm{B}} T}{R_{\text {shunt }}} \Delta f}$, where $k_{\mathrm{B}}$ is the Boltzmann constant and $T$ is the absolute temperature. To examine the influence of electrical noise on sensitive EQE measurements under electrical and light bias as well as under the open-circuit condition, PCE12:ITIC, PCDTBT:PCBM, and PBTTT:PCBM solar cells were measured at different applied bias voltages and (pump) light intensities. Details of the materials, device fabrication, and the current voltage performance of the organic solar cells are provided in the Supporting Information. We note that we have chosen two conventional bulk heterojunction polymer/fullerene systems PCDTBT:PCBM and PBTTT:PCBM) and one polymer/ nonfullerene system (PCE12:ITIC); the so-called nonfullerene acceptors (NFA) are the latest advance in organic photovoltaics and generate hitherto unrealised efficiencies $>16 \%$.
Figure 4a shows the NSD of the PCE12:ITIC solar cell measured at a frequency of $273 \mathrm{~Hz}$ and an electrical bandwidth of $1 \mathrm{~Hz}$ plotted as a function of applied bias. All NSD measurements were performed in the dark using a Spectrum Analyzer and different bias voltages between -1.5 and $0.85 \mathrm{~V}$ were applied using the internal, low noise voltage source of the preamplifier. The corresponding frequency dependent NSD spectra are provided in Figure $\mathrm{S} 1 \mathrm{~b}$ in the Supporting Information. The horizontal dashed line in Figure 4a marks the NSD of the preamplifier (at the highest gain of $10^{9} \mathrm{VA}^{-1}$ ) of approximately $1.62 \times 10^{-15} \mathrm{AHz}^{-1 / 2}$ and, therefore, represents the noise floor of the EQE apparatus. At zero bias (short-circuit) the thermal-noise-limited NSD of the PCE12:ITIC solar cell is approximately $1.36 \times 10^{-12}$ $\mathrm{AHz}^{-1 / 2}$. Here, the thermal noise due to the finite shunt resistance of the device leads to an increased NSD of approximately 3 orders of magnitude above the apparatus noise limit. The (thermal) noise current (limit) of the device can also be estimated from the shunt resistance of the device (see Supporting Information). As shown in Figure 4a, when increasing the forward or reverse bias, the electrical noise of the PCE12:ITIC solar cell rapidly increases by almost 3 orders of magnitude to roughly $10^{-9} \mathrm{AHz}^{-1 / 2}$ at an applied reverse bias of $-1.5 \mathrm{~V}$, respectively $0.85 \mathrm{~V}$. This is due to the shot noise induced by the bias current. Figure $4 b, c$ shows the experimentally obtained $\mathrm{EQE}_{\exp }$ (solid lines) and the corresponding noise floors (dotted lines corresponding to the $\mathrm{EQE}_{\mathrm{NE}}$ spectra) of the PCE12:ITIC solar cell measured over a broad range of wavelengths at different applied reverse and forward bias voltages. As shown in Figure $4 b, c$, the voltagedependent shot noise of the DUT strongly influences the sensitivity of the EQE measurements: while a minimum $\mathrm{EQE}_{\exp }$ at longer wavelengths of $10^{-7}$ at zero bias could be obtained (cf. black solid line in Figure 4b,c), only $10^{-4}$ (cf. blue solid line in Figure $4 \mathrm{~b}, \mathrm{c}$ ) could be achieved at an applied reverse bias voltage of $-1.5 \mathrm{~V}$, respectively forward bias of 0.85 $\mathrm{V}$. The noise floors shown by the dashed lines were obtained by blocking the monochromator beam. These values agree with the $\mathrm{EQE}_{\mathrm{NE}}$ spectra calculated from the noise currents of Figure 3a (see Supporting Information, Figure S3). This confirms, that the EQE is predominantly limited by the electrical shot noise under electrical bias and by the thermal noise at short circuit condition.

Sensitive EQE of Organic Solar Cells under Light Bias. In a similar manner, we further investigated the sensitive EQE under light bias conditions. Under these conditions, the overall noise current is limited by the thermal noise of the device and the photon shot noise of the light bias source with the latter being dominant at relevant light intensities up to 1-sunequivalent illumination. Figure $5 \mathrm{a}$ shows the NSD of the PCE12:ITIC solar cell as a function of short-circuit current density, $J_{s c}$. This is obtained by varying the power of three different bias light sources (a laser diode, an LED and a Halogen lamp). The dashed line represents the ideal NSD of the PCE12:ITIC solar cell as if it was light biased with a shotnoise-limited light source (ideal laser) with the photon noise of $\delta n=\sqrt{ }\langle n\rangle$, where $\langle n\rangle$ is the average photon number. We should note that an ideal laser is shot noise limited and clearly the laser used here is far from that limit. The $520 \mathrm{~nm}$ laser diode source exhibits the largest noise, which is approximately 3 orders of magnitude higher than the photon shot noise. The Halogen lamp exhibits the lowest noise and about $10 \times$ larger than the photon shot noise of the ideal coherent source. A 
a)

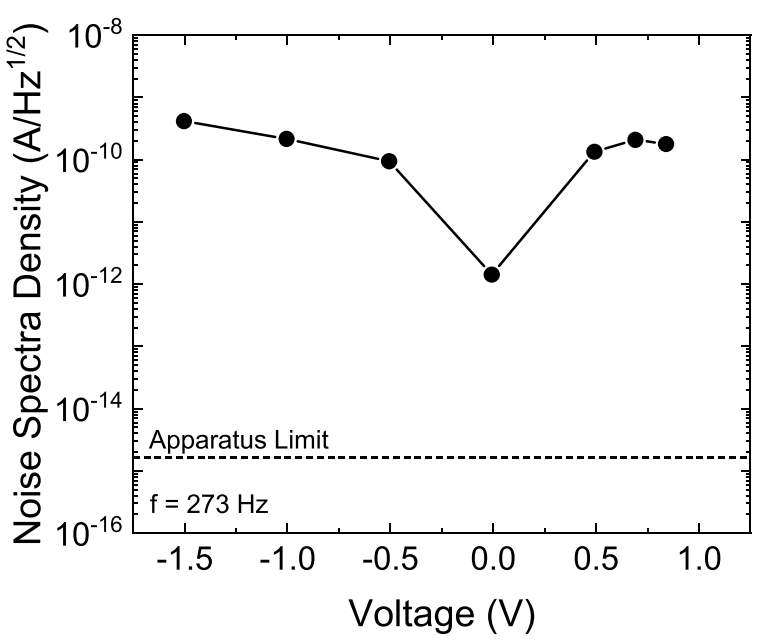

b)

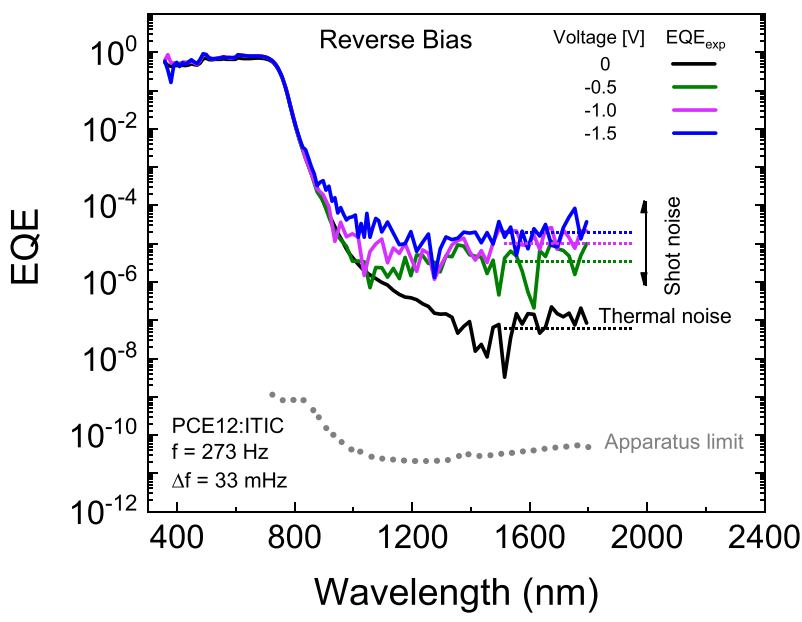

c)

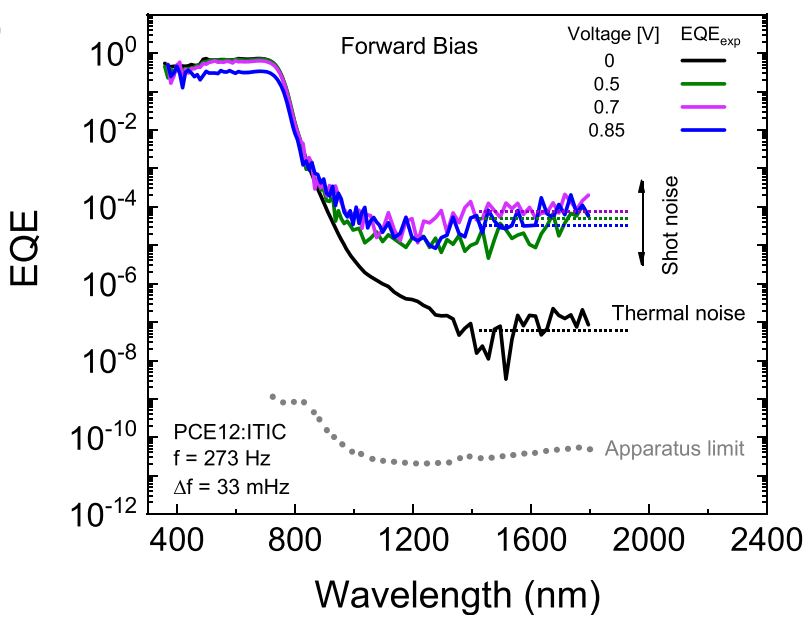

Figure 4. (a) Noise spectral density (NSD) of a PCE12:ITIC solar cell plotted as a function of the applied bias. Due to the finite shunt resistance of the device, the noise at zero bias (short-circuit) is larger than the apparatus noise floor (horizontal dashed line). Increasing the applied (forward or reverse) bias voltage results in a larger noise current due to the shot noise associated with the background DC current from the applied voltage. (b) External quantum efficiency (EQE) spectrum of a PCE12:ITIC solar cell measured at four different applied reverse biases (solid lines) and compared with the corresponding calculated noise equivalent $\mathrm{EQE}$ spectra $\left(\mathrm{EQE}_{\mathrm{NE}}\right.$; dotted lines) for each bias. While at zero bias a minimum EQE of approximately $10^{-8}$ could be achieved, the bias-dependent shot noise leads to a minimum EQE of only $10^{-4}$ at an applied voltage of $-1.5 \mathrm{~V}$. (c) Repetition of (b) but under different applied forward biases. thermal light source exhibits super-Poissonian photon statistics hence photon noise larger than the shot noise is expected. Based upon the measured noise currents under illumination we calculated the $\mathrm{EQE}_{\mathrm{NE}}$ spectra for our three different light sources (frequency dependent NSDs spectra are provided in Figure S4 in Supporting Information).

The results for the calculated $\mathrm{EQE}_{\mathrm{NE}}$ spectra under light bias with different light sources are shown in Figure $5 \mathrm{~b}$. The dotted line represents the expected $\mathrm{EQE}_{\mathrm{NE}}$ for the PCE12:ITIC solar cell when a shot-noise-limited light source is used. Among the three light sources, the halogen lamp exhibits the lowest noise thus lower $\mathrm{EQE}_{\mathrm{NE}}$. Therefore, for the rest of our experiment we employed this light source. Here we should emphasize the importance of the choice of the bias light source for measuring light-biased sensitive EQEs. A thermal light source such as a halogen lamp is often accessible while low noise (shot noise limited) lasers with high enough power are rather expensive. By using an intensity-squeezed light source where the photon statistics are sub-Poissonian (hence, photon noise below the shot noise), the $\mathrm{EQE}_{\mathrm{NE}}$ can be further reduced. However, such sources are again expensive and difficult to achieve high enough powers for light biasing. By decreasing the electrical bandwidth, similar improvements can be achieved, but this of course makes the measurement slower.

Figure $5 c$ shows the $\mathrm{EQE}_{\exp }$ (solid lines) spectra of a PCE12:ITIC solar cell measured at zero applied bias voltage (short-circuit) for the different bias powers corresponding to a range from 0 to 1 sun. The dashed lines indicate the $\mathrm{EQE}_{\mathrm{NE}}$. The $\mathrm{EQE}_{\text {exp }}$ strongly depends on the shot noise of the solar cell (Figure 5a) induced by the DC illumination and limits the sensitivity: while a minimum $\mathrm{EQE}_{\exp }$ at high wavelengths of approximately $10^{-7}$ could be obtained without additional illumination (cf. black solid line in Figure 5c), only $10^{-4}$ (cf. blue solid line in Figure 5c) could be achieved at a 1 sun equivalent output power. The calculated $\mathrm{EQE}_{\mathrm{NE}}$ again confirms the strongly shot noise limited EQE spectra (cf. dotted lines in Figure 5a). For the measurement of the EQE sensitively under the open-circuit condition, a high impedance voltage preamplifier was used (cf. Figure 1).

Figure $5 \mathrm{~d}$ shows the $\mathrm{EQE}_{\text {exp }}$ of the PCE12:ITIC solar cell measured under 1 sun equivalent DC output power of the halogen lamp both under short- and open-circuit conditions, and compared with the EQE spectrum obtained at zero bias without bias illumination. The horizontal dashed lines mark the corresponding noise floors at higher wavelengths. In this case the $\mathrm{EQE} E_{\exp }$ obtained under the open-circuit condition is almost 4 orders of magnitude lower than the $E Q E_{\exp }$ measured at short-circuit. Normalization of the $\mathrm{EQE}_{\exp }$ spectra (cf. Figure S5 in the Supporting Information) reveals no significant change in the spectral shape of the contribution of intra- and intermolecular states to the EQE between shortand open-circuit conditions.

Finally, we expanded upon the applicability of the above methodology to the other two model organic solar cell systems. Figure 6 compares EQE measurements performed on PBTTT:PCBM and PCDTBT:PCBM solar cells under both electrical (cf. Figure 6a,c) and light bias (cf. Figure 6b,d), including measurements of the EQE sensitively under the open-circuit condition. The current voltage performance under dark and artificial AM 1.5G illumination of the PBTTT:PCBM and PCDTBT:PCBM devices together with details of fabrication are provided in the Supporting Information (see Figure S2). All EQE measurements were performed at a 
a)

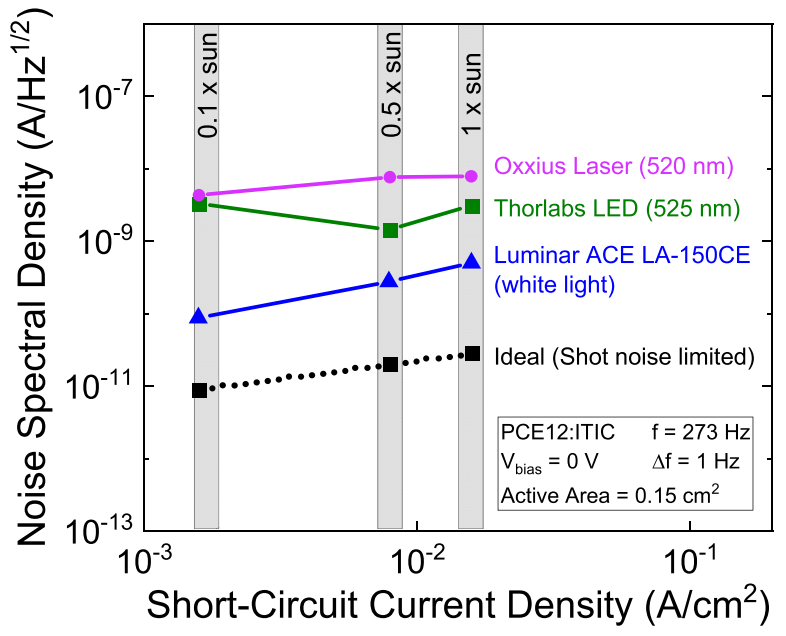

c)

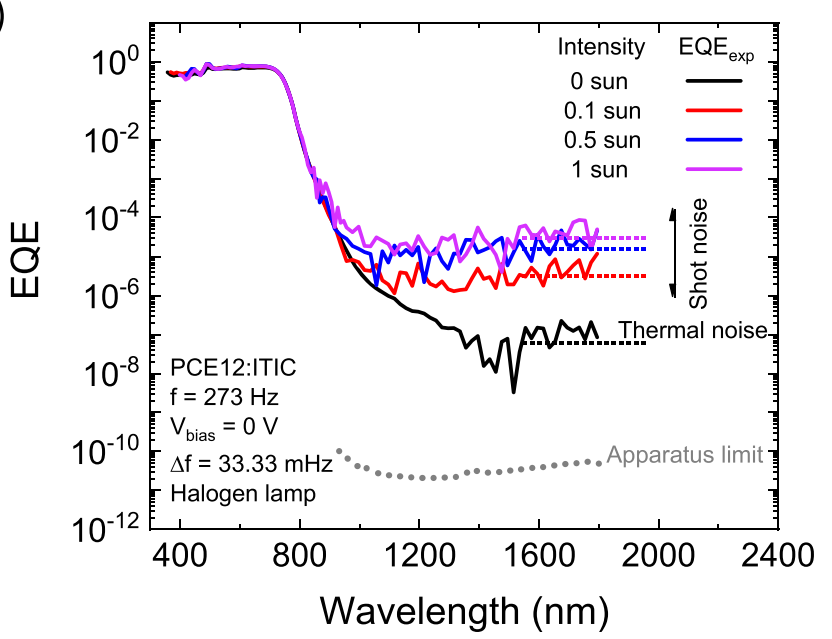

b)

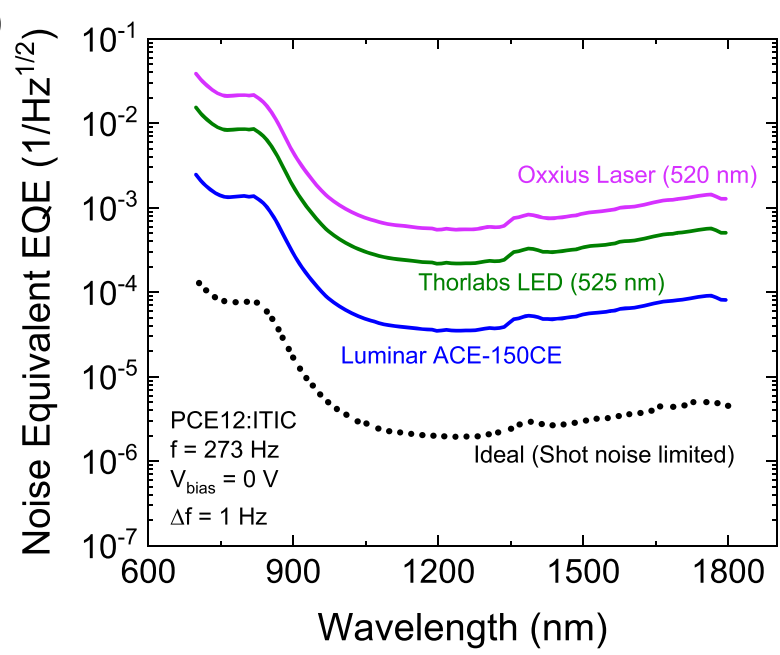

d)

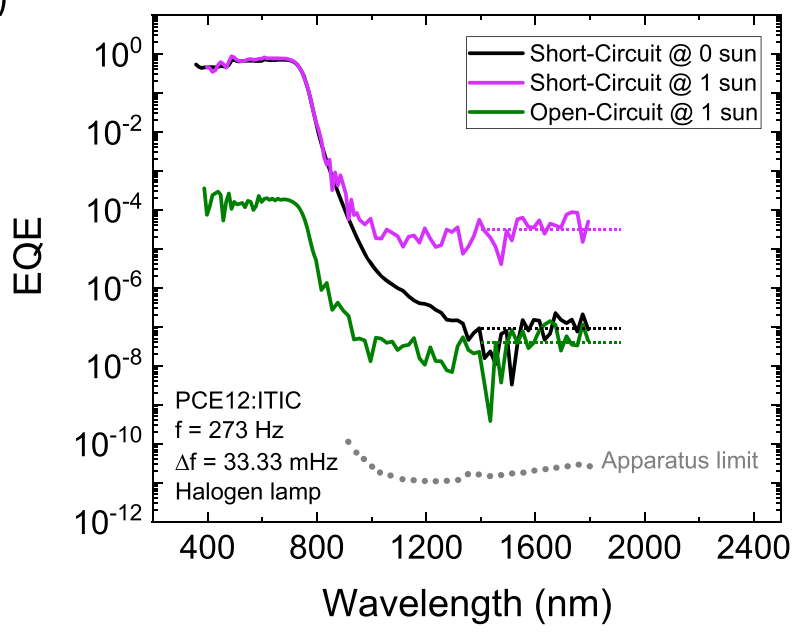

Figure 5. (a) Noise spectral density (NSD) of a PCE12:ITIC solar cell (at short-circuit) plotted as a function of short-circuit current density, and compared for the three different light sources. Increasing the pump light intensity (the short-circuit current density of the DUT) results in a larger noise current due to the shot noise associated with the DC background current. (b) Calculated noise equivalent external quantum efficiency $\left(\mathrm{EQE}_{\mathrm{NE}}\right)$ spectra under constant 1 sun equivalent pump intensity and compared for three different light sources. The dotted black line represents the ideal noise equivalent EQE which can be only achieved when a shot-noise-limited light source (ideal laser) is used for light biasing. The $\mathrm{EQE}_{\mathrm{NE}}$ (representing the minimum detectable EQE, normalized to the electrical bandwidth) strongly depends on the (optical) noise of the pump light source. (c) External quantum efficiency (EQE) spectrum of a PCE12:ITIC solar cell measured for three different white light halogen pump

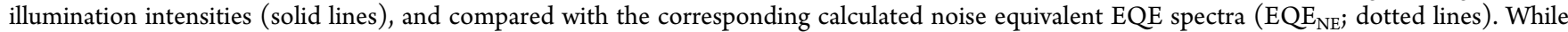
at zero pump illumination intensity a minimum EQE of approximately $10^{-8}$ could be achieved, the light bias-dependent shot noise leads to a minimum EQE of only $10^{-4}$ at 1 sun equivalent illumination. (d) EQE spectrum of a PCE12:ITIC solar cell under constant 1 sun equivalent pump intensity measured under short-circuit and open-circuit and compared with the EQE spectrum at zero pump intensity (at short-circuit).

frequency of $273 \mathrm{~Hz}$ and an electrical bandwidth of 33.33 $\mathrm{mHz}$.

The horizontal dashed lines mark the noise floors at high wavelengths. In both PBTTT:PCBM and PCDTBT:PCBM devices a tail in the EQE can be clearly resolved at energies well below the charge transfer states as indicated by the arrows in Figure 6a,c. The observed tail in the EQE at photon energies well below the charge transfer state energy indicates the presence of deep trap states with a spectral shape affected by the (micro-) cavity interference. ${ }^{25}$ This tail was also observed for the PCE12:ITIC in Figure 3. Under light bias for both short circuit and open-circuit, as shown in Figure 6a, the EQE of the PBTTT:PCBM solar cell can be still determined sensitively enough to obtain the charge transfer state absorption peak in the range of 800-1200 nm. No significant change in the spectral shape of the CT states is observed under electrical and/or light bias. Due to the photon shot noise, the tail of the subgab (deep traps) absorption feature can no longer be resolved when light bias is applied. Similar results were obtained for the PCDTBT:PCBM system and shown in Figure 6c,d.

\section{CONCLUSIONS}

In conclusion, in this contribution we have addressed the question of sensitive EQE measurements under light and electrical bias as well as open-circuit condition from a device and apparatus perspective with particular focus on the detection of weak photocurrent signals smaller than a fA at photon energies well below the bandgap. We showed that both optical and electrical noise can strongly limit the sensitivity of the EQE measurements. Optical noise (e.g., parasitic stray light) can in the first instance be reduced by the use of additional long pass filters to eliminate parasitic stray light or harmonics of the light source (spectrometer monochromator 
a)

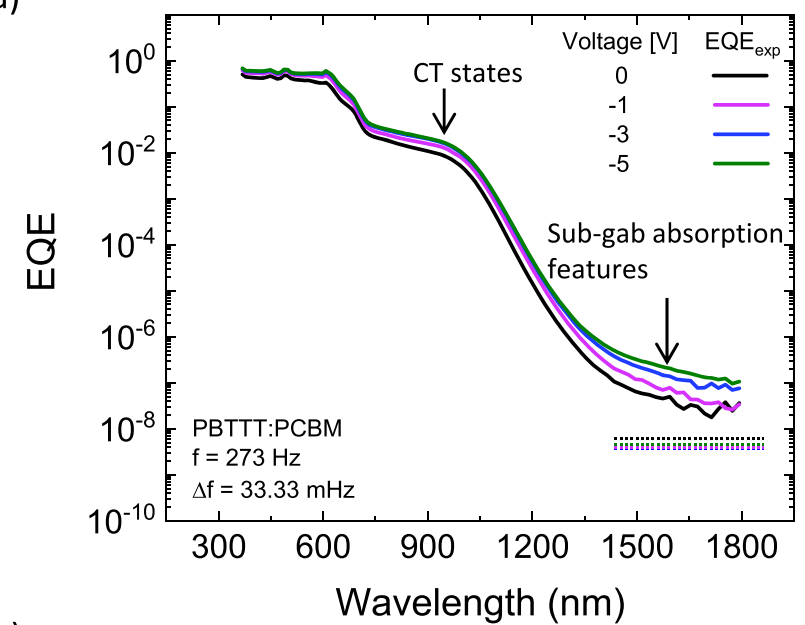

c)

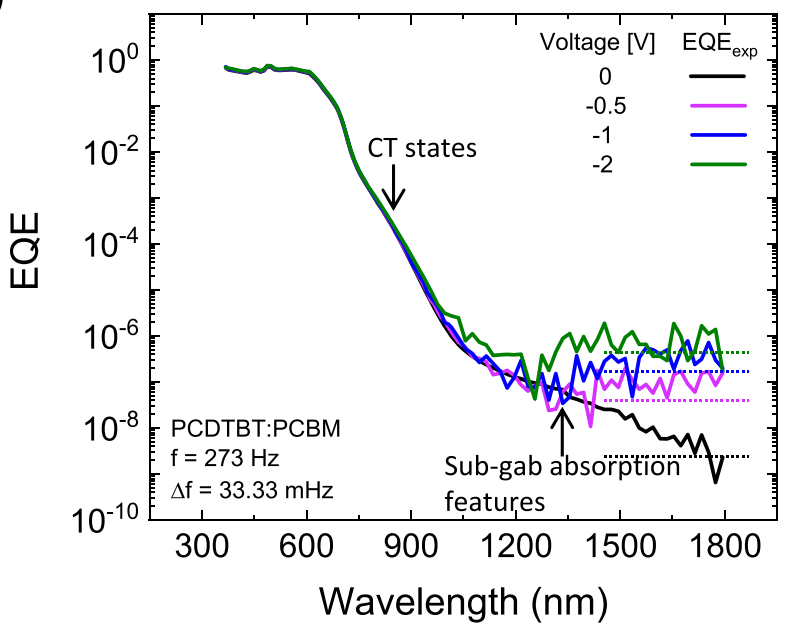

b)

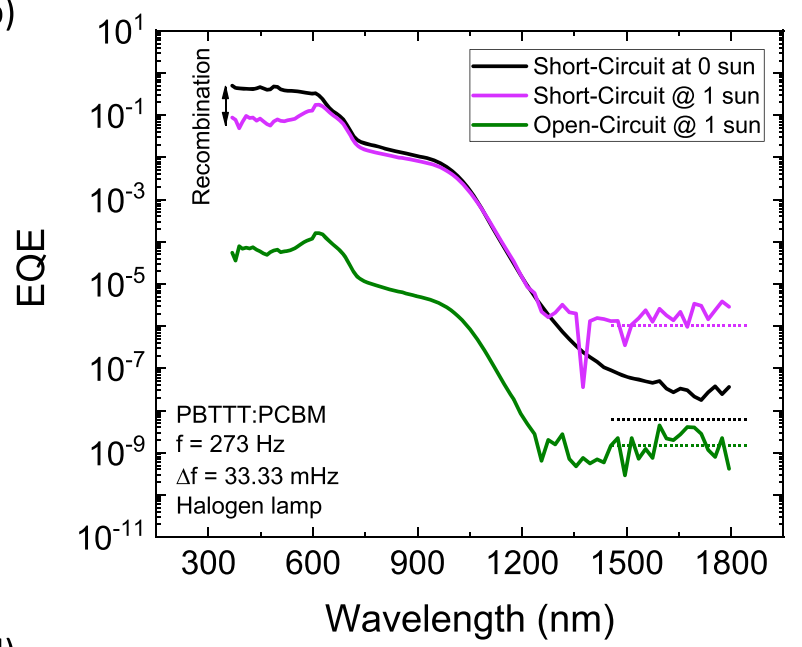

d)

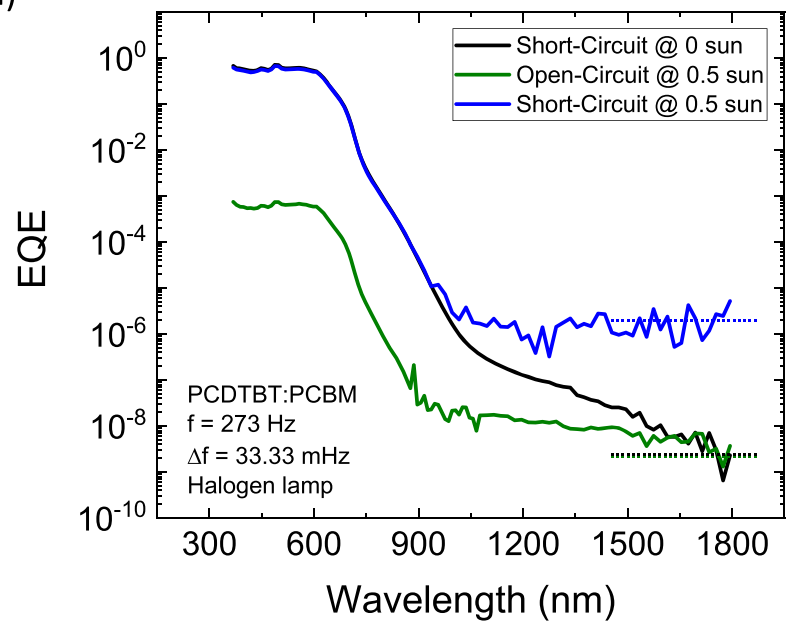

Figure 6. (a) External quantum efficiency (EQE) spectra of a PBTTT:PCBM solar cell measured for three different applied reverse bias voltages (solid lines). The dashed horizontal lines mark the corresponding noise floor. While at zero bias (short-circuit) a minimum EQE of approximately $10^{-8}$ could be achieved, the voltage dependent shot noise of the DUT leads to a minimum EQE of only $10^{-7}$ at an applied bias voltage of $-5 \mathrm{~V}$. (b) EQE spectrum of a PBTTT:PCBM solar cell under constant 1 sun equivalent pump intensity measured at short-circuit and open-circuit, and compared with the EQE spectrum at zero pump intensity (at short-circuit). Higher-order recombination of photogenerated charge carriers leads to a drop in EQE in the visible wavelength regime. (c) Repetition of panel (a) plotted for a PCTBT:PCBM solar cell measured at three different applied bias voltages. (d) Repetition of panel (c) but plotted for a PCDTBT:PCBM solar cell under 0.5 sun equivalent pump intensity.

system). The electrical noise, on the other hand, depends on both the EQE measurement system (e.g., preamplifier and wiring), the DUT (e.g., thermal or shot noise), and (in case of $\mathrm{EQE}$ under light bias) on the photon noise of the bias light source.

In a broader sense, this work demonstrates how a relatively simple direct photocurrent measurement methodology can be optimized to reliably measure EQEs as low as $10^{-10}$ and to observe subgap absorption features at photon energies well below the bandgap in both inorganic and organic optoelectronic devices. This has considerable utility for probing subgap states and is therefore of great importance for a better understanding of the nature of inter- and intramolecular states in organic and trap states in inorganic and organic solar cell and photodetectors.

\section{ASSOCIATED CONTENT}

\section{S Supporting Information}

The Supporting Information is available free of charge at https://pubs.acs.org/doi/10.1021/acsphotonics.9b01531.
Detailed description of device fabrication; current voltage performance of different organic solar cells in dark and under artificial 1 sun AM1.5 G condition; frequency-dependent noise spectral density spectra of different organic solar cells under electrical and light bias; experimental and calculated (noise equivalent) external quantum efficiency spectra of different organic solar cells under electrical and light bias; calculated (thermal) noise current (limit) based on the shunt resistance (PDF)

\section{AUTHOR INFORMATION}

\section{Corresponding Author}

*E-mail: ardalan.armin@swansea.ac.uk.

ORCID

Ardalan Armin: 0000-0002-6129-5354

Notes

The authors declare no competing financial interest. 


\section{ACKNOWLEDGMENTS}

The work was supported by the Sêr Cymru II Program through the European Regional Development Fund, Welsh European Funding Office, and Swansea University strategic initiative in Sustainable Advanced Materials. A.A. is a Sêr Cymru II Rising Star Fellow and P.M.is a Sêr Cymru II National Research Chair. S.Z. and C.K. are recipients of an EPSRC Doctoral Training Partnership studentship.

\section{REFERENCES}

(1) Sutter-Fella, C. M.; Miller, D. W.; Ngo, Q. P.; Roe, E. T.; Toma, F. M.; Sharp, I. D.; Lonergan, M. C.; Javey, A. Band Tailing and Deep Defect States in $\mathrm{CH} 3 \mathrm{NH} 3 \mathrm{~Pb}(\mathrm{I1}-\mathrm{XBrx}) 3$ Perovskites as Revealed by Sub-Bandgap Photocurrent. ACS Energy Lett. 2017, 2 (3), 709-715.

(2) Miller, D. W.; Eperon, G. E.; Roe, E. T.; Warren, C. W.; Snaith, H. J.; Lonergan, M. C. Defect States in Perovskite Solar Cells Associated with Hysteresis and Performance. Appl. Phys. Lett. 2016, 109 (15), 153902.

(3) Yao, J.; Kirchartz, T.; Vezie, M. S.; Faist, M. A.; Gong, W.; He, Z.; Wu, H.; Troughton, J.; Watson, T.; Bryant, D.; et al. Quantifying Losses in Open-Circuit Voltage in Solution-Processable Solar Cells. Phys. Rev. Appl. 2015, 4 (1), 1-10.

(4) Menke, S. M.; Ran, N. A.; Bazan, G. C.; Friend, R. H. Understanding Energy Loss in Organic Solar Cells: Toward a New Efficiency Regime. Joule 2018, 2 (1), 25-35.

(5) Hood, S.; Zarrabi, N.; Meredith, P.; Kassal, I.; Armin, A. Measuring Energetic Disorder in Organic Semiconductors Using the Photogenerated Charge-Separation Efficiency. J. Phys. Chem. Lett. 2019, 10, 3863-3870.

(6) Vandewal, K.; Tvingstedt, K.; Gadisa, A.; Inganäs, O.; Manca, J. V. Relating the Open-Circuit Voltage to Interface Molecular Properties of Donor:Acceptor Bulk Heterojunction Solar Cells. Phys. Rev. B: Condens. Matter Mater. Phys. 2010, 81 (12), 1-8.

(7) Vandewal, K.; Albrecht, S.; Hoke, E. T.; Graham, K. R.; Widmer, J.; Douglas, J. D.; Schubert, M.; Mateker, W. R.; Bloking, J. T.; Burkhard, G. F.; et al. Efficient Charge Generation by Relaxed Charge-Transfer States at Organic Interfaces. Nat. Mater. 2014, 13 (1), 63-68.

(8) Vandewal, K.; Tvingstedt, K.; Gadisa, A.; Inganäs, O.; Manca, J. V. On the Origin of the Open-Circuit Voltage of Polymer-Fullerene Solar Cells. Nat. Mater. 2009, 8 (11), 904-909.

(9) Pisoni, S.; Stolterfoht, M.; Löckinger, J.; Moser, T.; Jiang, Y.; Caprioglio, P.; Neher, D.; Buecheler, S.; Tiwari, A. N. On the Origin of Open-Circuit Voltage Losses in Flexible n-i-p Perovskite Solar Cells. Sci. Technol. Adv. Mater. 2019, 20 (1), 786-795.

(10) Vandewal, K.; Goris, L.; Haeldermans, I.; Nesládek, M.; Haenen, K.; Wagner, P.; Manca, J. V. Fourier-Transform Photocurrent Spectroscopy for a Fast and Highly Sensitive Spectral Characterization of Organic and Hybrid Solar Cells. Thin Solid Films 2008, 516 (20), 7135-7138.

(11) Chen, S.; Wang, Y.; Zhang, L.; Zhao, J.; Chen, Y.; Zhu, D.; Yao, H.; Zhang, G.; Ma, W.; Friend, R. H.; et al. Efficient Nonfullerene Organic Solar Cells with Small Driving Forces for Both Hole and Electron Transfer. Adv. Mater. 2018, 30 (45), 1-7.

(12) Vandewal, K.; Gadisa, A.; Oosterbaan, W. D.; Bertho, S.; Banishoeib, F.; Van Severen, I.; Lutsen, L.; Cleij, T. J.; Vanderzande, D.; Manca, J. V. The Relation between Open-Circuit Voltage and the Onset of Photocurrent Generation by Charge-Transfer Absorption in Polymer: Fullerene Bulk Heterojunction Solar Cells. Adv. Funct. Mater. 2008, 18 (14), 2064-2070.

(13) Ullbrich, S.; Benduhn, J.; Jia, X.; Nikolis, V. C.; Tvingstedt, K.; Piersimoni, F.; Roland, S.; Liu, Y.; Wu, J.; Fischer, A.; et al. Emissive and Charge-Generating Donor-Acceptor Interfaces for Organic Optoelectronics with Low Voltage Losses. Nat. Mater. 2019, 18 (5), 459-464.

(14) Kurpiers, J.; Ferron, T.; Roland, S.; Jakoby, M.; Thiede, T.; Jaiser, F.; Albrecht, S.; Janietz, S.; Collins, B. A.; Howard, I. A.
Probing the Pathways of Free Charge Generation in Organic Bulk Heterojunction Solar Cells. Nat. Commun. 2018, 9 (1), na.

(15) Azzouzi, M.; Yan, J.; Kirchartz, T.; Liu, K.; Wang, J.; Wu, H.; Nelson, J. Nonradiative Energy Losses in Bulk-Heterojunction Organic Photovoltaics. Phys. Rev. X 2018, 8 (3), 31055.

(16) Khan, S.-U.-Z.; Londi, G.; Liu, X.; Fusella, M. A.; D’Avino, G.; Muccioli, L.; Brigeman, A. N.; Niesen, B.; Yang, T. C.-J.; Olivier, Y.; et al. Multiple Charge Transfer States in Donor-Acceptor Heterojunctions with Large Frontier Orbital Energy Offsets. Chem. Mater. 2019, 31, 6808 .

(17) Yazmaciyan, A.; Meredith, P.; Armin, A. Cavity Enhanced Organic Photodiodes with Charge Collection Narrowing. Adv. Opt. Mater. 2019, 7 (8), 1-8.

(18) García De Arquer, F. P.; Armin, A.; Meredith, P.; Sargent, E. H. Solution-Processed Semiconductors for next-Generation Photodetectors. Nat. Rev. Mater. 2017, 2 (3), 1-16.

(19) Guo, F.; Yang, B.; Yuan, Y.; Xiao, Z.; Dong, Q.; Bi, Y.; Huang, J. A Nanocomposite Ultraviolet Photodetector Based on Interfacial Trap-Controlled Charge Injection. Nat. Nanotechnol. 2012, 7 (12), 798-802.

(20) Guo, F.; Xiao, Z.; Huang, J. Fullerene Photodetectors with a Linear Dynamic Range of 90 DB Enabled by a Cross-Linkable Buffer Layer. Adv. Opt. Mater. 2013, 1 (4), 289-294.

(21) Melskens, J.; Schouten, M.; Santbergen, R.; Fischer, M.; Vasudevan, R.; Van Der Vlies, D. J.; Quax, R. J. V.; Heirman, S. G. M.; Jäger, K.; Demontis, V.; et al. In Situ Manipulation of the Sub Gap States in Hydrogenated Amorphous Silicon Monitored by Advanced Application of Fourier Transform Photocurrent Spectroscopy. Sol. Energy Mater. Sol. Cells 2014, 129, 70-81.

(22) Collado-Fregoso, E.; Pugliese, S. N.; Wojcik, M.; Benduhn, J.; Bar-Or, E.; Perdigón Toro, L.; Hörmann, U.; Spoltore, D.; Vandewal, K.; Hodgkiss, J. M.; et al. Energy-Gap Law for Photocurrent Generation in Fullerene-Based Organic Solar Cells: The Case of Low-Donor-Content Blends. J. Am. Chem. Soc. 2019, 141 (6), 23292341.

(23) Armin, A.; Hambsch, M.; Kim, I. K.; Burn, P. L.; Meredith, P.; Namdas, E. B. Thick Junction Broadband Organic Photodiodes. Laser Photonics Rev. 2014, 8 (6), 924-932.

(24) Fang, Y.; Armin, A.; Meredith, P.; Huang, J. Accurate Characterization of Next-Generation Thin-Film Photodetectors. Nat. Photonics 2019, 13 (1), 1-4.

(25) Kaiser, C.; Zeiske, S.; Meredith, P.; Armin, A. Determining Ultralow Absorption Coefficients of Organic Semiconductors from the Sub-Bandgap Photovoltaic External Quantum Efficiency. Adv. Opt. Mater. 2019, 1901542. 